\title{
Intrapartum Antibiotics and Childhood Atopic Dermatitis
}

\author{
Debra L. Wohl, PhD, MS, William J. Curry, MD, MS, Dave Mauger, PhD, \\ Jennifer Miller, MD, and Kaitlyn Tyrie, BS
}

Introduction: Atopic dermatitis (AD) in children significantly impacts families because of medical costs, "lost" hours, and secondary characteristics such as asthma and ancillary infections. We investigate whether children delivered vaginally to women receiving intrapartum antibiotics have a greater risk of AD when younger than the age of 2 years than their counterparts.

Methods: We conducted a retrospective analysis of women who delivered child(ren) vaginally between 1996 and 2008. Women were identified as those who received intrapartum antibiotics and those who did not. Pediatric records were used to determine the incidence of AD.

Results: We collected data for 492 mother-child pairs. Intrapartum antibiotics were administered during 128 births; $28.9 \%$ of those children were diagnosed with AD by age 2 years (relative risk [RR], 1.03; 95\% confidence interval [CI], 0.75-1.41). Factors with the greatest risk of diagnosis of AD by 2 years of age were intrapartum antibiotic exposure for $>24$ hours (RR, 1.99; 95\% CI, 1.13-3.49), first born (RR, 1.78; 95\% CI, 1.33-2.38), and higher maternal education (RR, 1.43; 95\% CI, 0.99-2.06). No statistical differences in the prevalence of AD related to parental eczema, maternal group B Streptococcus status, or gestational age existed.

Conclusions: Exposure to antibiotics for $<24$ hours during a vaginal delivery does not increase the risk of $\mathrm{AD}$. Studies are needed to understand whether exposure for $>24$ hours during the intrapartum period increases the risk of AD. (J Am Board Fam Med 2015;28:82-89.)

Keywords: Antibiotics; Birth; Dermatitis, Atopic; Obstetrics; Pediatrics

The prevalence of atopic dermatitis $(\mathrm{AD})$ has been growing in industrialized countries over the past 40 years. ${ }^{1,2}$ Each year, AD results in significant medical costs, lost hours of schooling and employment, and increased mortality risk associated with secondary characteristics such as asthma, ancillary in-

This article was externally peer reviewed.

Submitted 16 January 2014; revised 30 May 2014; accepted 2 June 2014.

From the Department of Biology, Elizabethtown College, Elizabethtown, PA (DLW); the Department of Family and Community Medicine, Penn State Milton S. Hershey Medical Center, Hershey, PA (WJC); the Department of Public Health Sciences, Pennsylvania State University College of Medicine, Hershey (WJC, DM); the Department of Pediatrics, Penn State Milton S. Hershey Medical Center and Pennsylvania State University College of Medicine, Hershey (JM); and the Pennsylvania State University College of Medicine, Hershey (KT).

Funding: Financial support provided by National Institute of Health Award no. 1R15A1076933-01A1 (to DLW). Conflict of interest: none declared.

Corresponding author: Debra L. Wohl, PhD, Department of Biology, Elizabethtown College, 1 Alpha Hall, Elizabethtown, PA 17022 (E-mail: wohld@etown.edu). fections, and smallpox vaccination complications. ${ }^{3}$ Susceptibility genes, immunologic factors, the host environment, and skin barrier defects are associated with the development of $\mathrm{AD}$ but do not explain the increased prevalence. Current medical procedures may contribute by either directly or indirectly disrupting the host's microbiota. Microorganisms have key roles in a host's metabolic function (eg, vitamin and amino acid synthesis, bile and biotransformations), immune system development, pathogen resistance, angiogenesis, and fat storage. ${ }^{4-6}$ Intestinal microbiota and, consequently, fecal samples of children who have $\mathrm{AD}$, asthma, or allergies are distinctive from those of their nonatopic counterparts. ${ }^{7-10}$ Therefore antibiotic exposure disrupting the colonizing microbiota during the birthing process may lead to $\mathrm{AD}$.

The risk of $\mathrm{AD}$ in children younger than 2 years of age with antibiotic exposure in utero during the second and/or third trimester of pregnancy is elevated. ${ }^{11,12}$ Exposure to antibiotics during the first 
months of life significantly increase the odds of a later diagnosis of allergic disease. ${ }^{13-15}$ Negele et $\mathrm{al}^{16}$ examined data from 2500 healthy, full-term infants, of whom 435 were born by caesarean delivery and 2065 by vaginal delivery. Infants born by caesarean delivery have an elevated risk of developing asthma, $\mathrm{AD}$, or allergic rhinoconjunctivitis compared with their counterparts. ${ }^{17}$ Others report similar findings. ${ }^{15,17,18}$ Because $\mathrm{AD}$ is not characterized by a single marker but is rather a cluster of clinical findings and multiple immunologic abnormalities, it is often excluded from studies examining asthma and allergies. For example, a study by Hakansson and Kallen ${ }^{19}$ also showed cesarean delivery increased the relative risk for asthma and gastroenteritis in children, but it did not explore AD. The increase in the prevalence of $\mathrm{AD}$ may mirror clinical practices in Western societies, such as cesarean deliveries and antibiotic use. Currently, antibiotics are often prescribed to women during pregnancy for urinary tract infections, community-acquired pneumonia, chorioamnionitis, and sexually transmitted diseases. During labor and delivery women often receive antibiotics intravenously as prophylaxis for group b Streptococcus (GBS) and premature rupture of membranes. Antibiotics administered to the mother have been shown to disrupt her microflora. $^{20}$

Our primary study objective was to determine whether children delivered vaginally and exposed to intrapartum antibiotics have a greater risk of developing $\mathrm{AD}$ by the age of 2 years than their counterparts not exposed to antibiotics. Given the growing prevalence of $\mathrm{AD}$, we wanted to determine whether the indirect disruption of the colonizing microbiota by the administration of intrapartum antibiotics to the mother has the unintended longterm consequence of $\mathrm{AD}$. We tested the hypothesis that there is a significant positive relationship between the administration of antibiotics during a vaginal delivery and the development of $\mathrm{AD}$ in children younger than 2 years old. In our retrospective study we analyzed the medical records of women who gave birth vaginally and either received or did not receive intrapartum antibiotics and the medical records of their children.

\section{Methods}

Mothers of a child(ren) who was delivered vaginally without complications and was now between 2 and
10 years old were invited into the study. Potential participants were identified for the study in accordance with the Milton S. Hershey Medical Center's Human Subjects Protection Office Institutional Review Board (protocol no. 28656EP) and Elizabethtown College's Institutional Review Board (protocol no. 200846). Hospital billing records from the Milton S. Hershey Medical Center in south-central Pennsylvania were used to identify potential participants based on coding of a vaginal delivery. Women invited to the study received a short letter from their primary care provider that included an introduction to the study, the study aims, a description of the targeted population, and contact information should they have questions. A link to an electronic questionnaire and record release forms for the child's pediatric records, along with a description of how the data would be used and secured, were also included with the letter of invitation. Interested participants enrolled by completing and returning the medical release forms.

Data for each mother-child pair were abstracted from labor and delivery inpatient records, as well as the child's inpatient and outpatient records. In addition, participants were required to complete an electronic questionnaire (developed with Franklin and Marshall College's Center for Opinion Research). For those participants who were unable to complete the questionnaire online, options to use a computing center or a paper copy of the questionnaire were provided. The questionnaire was used to collect data on the pregnancy; delivery of the child; race/ethnicity; family history, including the presence of chronic illnesses or diseases that run in the family; and lifestyle choices (eg, smoker vs nonsmoker, breastfeeding and duration, pets). Information pertaining to family history of $\mathrm{AD}$, asthma, or allergies and whether the child has siblings with or without $\mathrm{AD}$, asthma, or allergies was collected. Participants were remunerated after completing the survey.

Participants were dichotomized as those who received intrapartum antibiotics and those who did not. Participants retained for the study included women who were older than 18 years of age at the time of invitation, those who had an uncomplicated vaginal delivery resulting in the birth of a child whose birth weight was between 2268 and 4753 g, and children not diagnosed with complications or congenital malformations during the perinatal or postdelivery period. Inpatient medical records of 
the mother were used to identify results of the GBS test, other pregnancy complications, the amount of time she spent in labor at the hospital, and information pertaining to the administration of antibiotics during delivery. Children's medical records were used to exclude children who did not meet the birth weight criteria, as well as children with a 5 -minute Apgar score $<5$. Data pertaining to any course of antibiotics the newborn received were recorded, as well as the duration of the child's hospital stay. The child's medical records also were used to identify the severity of AD and other measures of health based on records from well-baby visits. $\mathrm{AD}$ was considered present when there was a presumed history from parent with confirmation in the medical records or when a prescription drug classed for the treatment of eczema or $\mathrm{AD}$, such as pimecrolimus, tacrolimus, or topical corticosteroids, had been prescribed because of the presence of a pruritic rash.

Based on the conservative measure of the prevalence of $\mathrm{AD}$ at $15 \%$ among those not exposed to intrapartum antibiotics and 28\% for those exposed to intrapartum antibiotics, as well as an $80 \%$ power with an odds ratio of 1.6 , we estimated a need to enroll 594 women, of whom half were exposed to intrapartum antibiotics. Descriptive statistics including the mean, median, and standard deviation were used to characterize the study with respect to continuous measures and frequency tables to summarize those measures that are categorical. Relative risks and their $95 \%$ confidence intervals (CIs) were used to characterize the associations between $\mathrm{AD}$ and exposure to intrapartum antibiotics and the other covariates. Interaction effects between the primary exposure and the other covariates also were explored. Secondary analyses tested other specific aspects of the delivery and pregnancy, including a positive test for GBS during pregnancy, the type of antibiotic administered during labor and delivery, intrapartum antibiotic exposure at the hospital (classified as $\leq 4,4-12,12-24,>24$ hours), and receipt of antibiotics by the neonate. For analyses, antibiotics were grouped as penicillins, cephalosporins, macrolides, and aminoglycosides. In addition, some of the women who participated in the study had given birth to more than 1 child meeting the selection criteria. In such circumstances data corresponding to all eligible births was collected. Outcome data from siblings were correlated with each other because of their shared environmental conditions and genetic makeup. The proportion of sibling children (ie, single-pregnancy multiples or maternal siblings from separate pregnancies) who were included in the study was $26 \%$. Relative risks and associated CIs were estimated using log-linear models, and the generalized estimating equation approach was used to account for the lack of independence between siblings. All analyses were performed using SAS software version 9.2 (SAS Inc., Cary, NC).

\section{Results}

Invitations to participate in the study were mailed to 1143 women who had vaginal deliveries at Milton S. Hershey Medical Center between 1996 and 2008; $12 \%(n=137)$ of the invitations were returned by the US Postal Service. Respondents totaled 525 women, of whom $3.5 \%$ were not enrolled in the study because the medical release was incomplete.

A total of 507 women (and their child[ren]) were enrolled in the study. These women self-identified as Hispanic or Latino $(\mathrm{n}=8)$, Asian $(\mathrm{n}=11)$, black or African American ( $\mathrm{n}=11)$, American Indian/ Alaska Native $(\mathrm{n}=1)$, Native Hawaiian or other Pacific Islander $(n=1)$, white $(n=435)$, and more than one race $(n=2)$. The ethnicity/race was unknown for 38 women. Based on US Census records from Dauphin and Lancaster counties, the population was representative of the area (http:// www.census.gov/popfinder/).

We analyzed data from 492 mother-child pairs for which we were able to link hospital labor and delivery records to the child's pediatric records and the inclusion criteria were met. The mothers' mean age was $29.8 \pm 5.1$ years. Children enrolled in the study had a mean gestational age of $39.3 \pm 1.4$ weeks and a mean weight of $3507.0 \pm 473.7 \mathrm{~g} ; 228$ $(46.3 \%)$ were girls. Intrapartum antibiotics were administered during 128 births (Table 1). Penicillins were the most frequent class of drugs used ( $\mathrm{n}=$ 108), followed by macrolides $(\mathrm{n}=16)$, aminoglycosides $(\mathrm{n}=3)$, and cephalosporins $(\mathrm{n}=1)$. No significant differences between the intrapartum drug classes and the occurrence of $\mathrm{AD}$ were detected $(P=.0881)$. Of those women receiving intravenous antibiotics during delivery, 16 received more than 1 class of antibiotic. Intrapartum antibiotic exposure and diagnosis of $\mathrm{AD}$ by 2 years of age occurred in $28.9 \%$ of the children (relative risk [RR], 1.03; 95\% CI, 0.75-1.41). 
Table 1. Relative Risk of Atopic Dermatitis Associated With Infant Characteristics

\begin{tabular}{|c|c|c|c|}
\hline & Eczema, \% (n/N) & RR (95\% CI) & $P$ Value \\
\hline \multicolumn{4}{|l|}{ Sex } \\
\hline Male & $30.3(80 / 264)$ & $1.19(0.9-1.56)$ & .229 \\
\hline Female & $25.0(57 / 228)$ & - & - \\
\hline \multicolumn{4}{|l|}{ Intrapartum antibiotics } \\
\hline Yes & $28.9(37 / 128)$ & $1.03(0.75-1.41)$ & .854 \\
\hline No & $27.5(100 / 364)$ & - & - \\
\hline \multicolumn{4}{|l|}{ Birth order } \\
\hline First born & $36.9(80 / 217)$ & $1.78(1.33-2.38)$ & .0001 \\
\hline Second born or later & $20.7(57 / 275)$ & - & - \\
\hline \multicolumn{4}{|l|}{ Maternal eczema (ever)* } \\
\hline Yes & $32.3(21 / 65)$ & $1.16(0.77-1.74)$ & .495 \\
\hline No & $32.2(98 / 350)$ & - & - \\
\hline \multicolumn{4}{|l|}{ Paternal eczema (ever) } \\
\hline Yes & $41.4(12 / 29)$ & $1.55(0.96-2.51)$ & .146 \\
\hline No & $27.7(106 / 382)$ & - & - \\
\hline \multicolumn{4}{|l|}{ Sibling eczema (ever)* } \\
\hline Yes & $31.4(28 / 80)$ & $1.26(0.85-1.86)$ & .344 \\
\hline No & $27.3(81 / 311)$ & - & - \\
\hline \multicolumn{4}{|l|}{ Maternal asthma (ever)* } \\
\hline Yes & $27.9(19 / 68)$ & $0.99(0.65-1.49)$ & .958 \\
\hline No & $28.6(99 / 346)$ & - & - \\
\hline \multicolumn{4}{|l|}{ Paternal asthma (ever) ${ }^{*}$} \\
\hline Yes & $34.1(14 / 41)$ & $1.20(0.75-1.93)$ & .477 \\
\hline No & $28.1(104 / 370)$ & - & - \\
\hline \multicolumn{4}{|l|}{ Pets (cats/dogs)* } \\
\hline Yes & $29.0(80 / 276)$ & $1.10(0.73-1.66)$ & .652 \\
\hline No & $27.4(26 / 95)$ & - & - \\
\hline \multicolumn{4}{|l|}{ GBS status } \\
\hline Negative & $63(63 / 229)$ & $1.32(0.83-2.09)$ & .200 \\
\hline Positive & $21.0(17 / 81)$ & - & - \\
\hline \multicolumn{4}{|l|}{ Smoking* } \\
\hline No & $29.5(120 / 407)$ & $1.65(0.66-4.13)$ & .207 \\
\hline Yes & $17.4(4 / 23)$ & - & - \\
\hline \multicolumn{4}{|c|}{ Infant feeding during first 3 months* } \\
\hline Primarily breastfed & $29.7(85 / 286)$ & $1.10(0.79-1.53)$ & .579 \\
\hline Primarily formula fed & $26.9(39 / 145)$ & - & - \\
\hline \multicolumn{4}{|l|}{ Gestational age (weeks) } \\
\hline$\leq 40$ & $27.2(94 / 346)$ & & \\
\hline$>40$ & $30.1(43 / 143)$ & $1.07(0.78-1.46)$ & .678 \\
\hline \multicolumn{4}{|l|}{ Birth weight (grams) } \\
\hline$<2500$ & $57.1(4 / 7)$ & $4.23(0.9-19.92)$ & .068 \\
\hline $2500-4500$ & $27.7(132 / 477)$ & $1.92(0.96-3.83)$ & .064 \\
\hline$>4500$ & $12.5(1 / 8)$ & - & - \\
\hline \multicolumn{4}{|l|}{ Maternal education (years)* } \\
\hline Did not attend college & $22.5(32 / 142)$ & $0.70(0.48-1.01)$ & .039 \\
\hline College degree or more & $32.0(97 / 303)$ & - & - \\
\hline
\end{tabular}

${ }^{*}$ Self-reported.

$\mathrm{CI}$, confidence interval; RR, relative risk. 
Table 2. Intrapartum Antibiotic Exposure by Time and Atopic Dermatitis* $(n=118)$

\begin{tabular}{llll}
\hline $\begin{array}{l}\text { Exposure } \\
\begin{array}{l}\text { Time } \\
\text { (hours) }\end{array}\end{array}$ & $\begin{array}{l}\text { Eczema, } \\
\%(\mathrm{n} / \mathrm{N})\end{array}$ & $\mathrm{RR}^{\dagger}(95 \% \mathrm{CI})$ & $\begin{array}{c}P \\
\text { Value }\end{array}$ \\
\hline $0-4$ & $32.1(9 / 28)$ & $1.17(0.66-2.06)$ & .59 \\
$4-12$ & $20.8(11 / 53)$ & $0.76(0.44-1.31)$ & .319 \\
$12-24$ & $26.9(7 / 26)$ & $0.98(0.51-1.89)$ & .952 \\
$>24$ & $54.5(6 / 11)$ & $1.99(1.13-3.49)$ & .017 \\
\hline
\end{tabular}

*Atopic dermatitis was diagnosed in 10 children who were not included in the analyses because intrapartum antibiotic exposure time could not be determined.

${ }^{\dagger}$ Relative risks (RRs) were calculated when compared with no intrapartum exposure $(n=364)$ and atopic dermatitis $(n=100)$. $\mathrm{CI}$, confidence interval.

As shown in Tables 1 and 2, the greatest risks of being diagnosed with $\mathrm{AD}$ by 2 years of age were associated with intrapartum antibiotic exposure for $>24$ hours (RR, 1.99; 95\% CI, 1.13-3.49), first born (RR, 1.78; 95\% CI, 1.33-2.38), and higher maternal education (RR, 1.43; 95\% CI, 0.99-2.06). The RR between childhood eczema and familial history of eczema was not statistically significant (mother: RR, 1.16; 95\% CI, 0.77-1.74; father: RR, 1.55; 95\% CI, 0.96-2.51; siblings: RR, 1.26; 95\% CI, $0.85-1.86)$. It was also not statistically significant between childhood eczema and familial history of asthma (mother: RR, 0.99; 95\% CI, 0.65-1.54; father: RR, 1.20; 95\% CI, 0.75-1.93; siblings: RR, 1.07 ; $95 \% \mathrm{CI}, 0.67-1.75$ ) or allergies (mother: RR, 0.93; 95\% CI, 0.68-1.25; father: RR, 1.23; 95\% CI, 0.92-1.64; siblings: RR, 0.85; 95\% CI, 0.60-1.22). There was also no significant relationship with sex, pets, or maternal cigarette use (Table 1). Women who tested positive for GBS comprised a subset of the women who received intrapartum antibiotics ( $\mathrm{n}=81 ; 16.4 \%$ ); no significant risk of $\mathrm{AD}$ was associated with testing positive for GBS (RR, 0.76; 95\% CI, 0.48-1.20; Table 1).

Exposure to antibiotics as a neonate did not increase the relative risk of developing $\mathrm{AD}$ (Table 3). Within the first 24 hours of delivery, 31 of the children in the study had received antibiotics. By 2 years of age, 348 of the 492 children had received at least 1 dose of antibiotics; 103 of the children (23.6\%) were diagnosed with $\mathrm{AD}$ (RR, 1.26; $95 \%$ CI, 0.90-1.77). Breast milk versus formula feedings did not change the risk of $\mathrm{AD} ; 39$ of 145 infants fed primarily formula for the first 3 months of life developed $\mathrm{AD}$, whereas 85 of 286 infants receiving breast milk in the first 3 months developed AD (Table 1).

\section{Discussion}

We found intrapartum antibiotic exposure for $>24$ hours may increase the likelihood that children younger than the age of 2 will develop AD. In our study, 11 children were exposed to intrapartum antibiotics for $>24$ hours, and none of their mothers had tested positive for GBS, yet 6 of them developed $\mathrm{AD}$. While most research has focused on antibiotic exposure during the prenatal or postpartum periods and mode of delivery, antibiotic exposure during the intrapartum period alters the mother's microbiome. ${ }^{21,22}$ Although intrapartum exposure to antibiotics for $<24$ hours was not statistically significant (Table 2), extended exposure to antibiotics $>24$ hours during the intrapartum period may increase the risk of $\mathrm{AD}$ due to disruption of microbial colonization or other factors such as environmental exposures or genetic predisposition and merits additional study. We also found a significant RR of diagnosis of $\mathrm{AD}$ in children younger than age 2 associated with birth order and maternal education; first-born children or children of mothers who have a college education or higher both increased the likelihood that a child would be diagnosed with $\mathrm{AD}$ (Table 1).

The extensive use of antibiotics in general medicine, and particularly during the critical time of

Table 3. Relative Risk of Atopic Dermatitis Associated With Infant Exposure to Antibiotics

\begin{tabular}{lccc}
\hline $\begin{array}{l}\text { Age at Exposure } \\
\text { to Antibiotics }\end{array}$ & Eczema, \% (n/N) & RR (95\% CI) & $\begin{array}{c}P \\
\text { Value }\end{array}$ \\
\hline$<24$ Hours & & & \\
$\quad$ Yes & $38.7(12 / 32)$ & $1.43(0.92-2.24)$ & .186 \\
$\quad$ No & $27.1(125 / 461)$ & - & - \\
$<7$ Days & & & \\
Yes & $35.9(14 / 39)$ & $1.32(0.88-1.99)$ & .245 \\
No & $27.2(123 / 453)$ & - & - \\
$<30$ Days & & $-11(0.74-1.67)$ & .627 \\
Yes & $30.5(18 / 59)$ & - & - \\
$\quad$ No & $27.5(119 / 433)$ & & \\
$<1$ Year & & - & - \\
Yes & $30.2(80 / 265)$ & $1.19(0.89-1.59)$ & .235 \\
$\quad$ No & $25.1(57 / 227)$ & - & - \\
By 2 years & & - & -163 \\
Yes & $23.6(103 / 348)$ & $1.26(0.9-1.77)$ & - \\
No & $23.6(34 / 144)$ & - & \\
\hline
\end{tabular}

$\mathrm{CI}$, confidence interval; RR, relative risk. 
birth, highlights the need for examination of medical practices that may contribute to unintended consequences. While the use of antibiotics is certainly associated with significant short-term medical gains, they may have unintended long-term consequences. This study controlled for method of delivery to focus on the disruption of microbial colonization of the neonate solely by the administration of intrapartum antibiotics. Examination of pediatric records and elective questionnaires were used to confirm AD. Earlier studies showed that disruption of microbial colonization in neonates may lead to the development of atopies, asthma, and allergies. ${ }^{12,21-24} \mathrm{Few}$, if any, studies, however, have controlled for both birthing method (ie, vaginal or caesarean delivery) and antibiotic exposure. Therefore the cause of early childhood AD remains unclear. Our retrospective examination provided the opportunity to examine vaginal deliveries and a single atopy, childhood eczema. We report RRs because the limited number of significant relationships did not warrant a logistic regression analysis. Self-reported variables such as familial history, pets, smoking, and sibling outcomes rely on recall and carry less significance than data from medical records. Self-reported data are, however, important when examining a multifaceted chronic disease such as $\mathrm{AD}$. Because initial microbial colonization can define successional trajectories ${ }^{25}$ and because microbiota are directly involved in the maturation of the immune system, ${ }^{26}$ microbial colonization of the neonate is a crucial time in development and should be examined to ensure short-term gains do not have unintended long-term consequences.

When women received intrapartum antibiotics, penicillins were administered $84.4 \%$ of the time; there was no difference in $\mathrm{AD}$ risk when analyzing penicillins versus other antibiotics. Exposure to antibiotics delivered intravenously for $>4$ hours can decrease vertical transmission of GBS and other early onset invasive infections. ${ }^{27,28}$ In our study the risk of $\mathrm{AD}$ was not elevated unless antibiotics had been administered for $>24$ hours. None of the 11 women receiving intrapartum antibiotics for $>24$ hours in our study tested positive for GBS, suggesting other factors may be important. Our hypothesis that intrapartum antibiotics and the subsequent disruption of colonizing microbiota in the neonate lead to increased risk of $\mathrm{AD}$ is supported. Direct exposure to antibiotics in children between birth and 2 years of age did not increase the risk of developing $\mathrm{AD}$. Interestingly, birth order and maternal education are both positively related with the likelihood a child will be diagnosed with $\mathrm{AD}$ before the age of 2 years (Table 1). Others found that first-born children have a greater likelihood of AD. ${ }^{29,30}$ Researchers have suggested the finding of first born and increased risk of $\mathrm{AD}$ is explained by the hygiene hypothesis, which assumes a lack of microbial antigen exposure results in underproduction of $T h_{1}$ and subsequent over production of $\mathrm{Th}_{2}$ cells. ${ }^{31}$ Although our study supports this finding, we believe it may also reflect a reporting bias. First-born children typically have the undivided attention of the caregiver and a symptomatic rash may lead to clinician visits. ${ }^{32}$ To fully explain first born and $\mathrm{AD}$ using the hygiene hypothesis, we would expect elevated RRs associated with the absence of pets and primarily being fed formula during the first 3 months of life reducing microbial exposures. These were not observed (Table 1). Furthermore, reporting bias may also account for the significant relationship of the mother's education and diagnosis of $\mathrm{AD}$. Older mothers and those with additional years of schooling beyond high school may be more likely to take their child to a clinician because of a symptomatic rash observed with $\mathrm{AD}$ and/or have more confidence to mention the condition during an office visit. ${ }^{32,33}$

Heritable mutations in the skin barrier gene filaggrin predispose children to $\mathrm{AD}$ and asthma. ${ }^{34}$ Our study supports $\mathrm{AD}$ as a multifactorial disease, and although genetic inheritance was not significant and does not explain the presence of $\mathrm{AD}$ in young children, it may be a contributing factor. Further examination of the role of genetics in $\mathrm{AD}$ is needed; in our study it may have been masked by the fact that all family history data were self-reported recall data, as well as the low power of our study.

Based on the finding that there is no significant relationship between the short-term use of intrapartum antibiotics and the development of early childhood eczema, we anticipate no clinical change in the administration of intrapartum antibiotics. However, we encourage clinicians to reevaluate possible short-term versus long-term risks to neonates with intrapartum antibiotic exposure for $>24$ hours because it possibly increases the risk of $\mathrm{AD}$. Although limited in size and power, our study reveals no unintended long-term atopic consequences associated with the short-term use of in- 
trapartum antibiotics. Intrapartum antibiotic use that directly benefits the neonate, such as prophylaxis for limiting the possible spread of GBS or sexually transmitted diseases, should continue because they outweigh long-term risks.

Limitations of our study included the inability to achieve our projected sample size, which reduced the power of the study, increasing the risk of a type II error. The retrospective nature of the study, relying on physicians' documentation reporting atopies and historic reporting by parents, could have led to underreporting of atopic disease, as well. The study was performed in a small area of south-central Pennsylvania and may not be generalizable to other populations. In conjunction, the lack of heterogeneity may also impact generalization.

An understanding of the increase in the prevalence of $\mathrm{AD}$ has not been provided by this study. $\mathrm{AD}$, a chronic disease, affects almost one-third of the population. ${ }^{2,35}$ Developing clear biomarkers may help reveal how genetics, microbial colonization, immune system development, and the environment factor in to the presence and prevalence of this disease. Further investigation should examine broader demographics, dietary exposures, and other environmental exposures in the first 2 years of life.

The authors thank Valerie Martin for early formulation; Janie Crow for organizing and coordinating access to hospital records; and Katie Diamond, Stephanie (Dougherty) Eastwick, Brittany Kuperavage, Muhammed Arslan Rashid, and Liesl Sieber for their involvement in data collection.

\section{References}

1. Warmer JO. Worldwide variations in the prevalence of atopic symptoms: what does it all mean? Thorax 2007;54:S46-51.

2. ISAAC. Worldwide variation in prevalence of symptoms of asthma, allergic rhinoconjunctivitis, and atopic eczema: ISAAC. The International Study of Asthma and Allergies in Childhood (ISAAC) Steering Committee. Lancet 1998;351:1225-32.

3. Williams HC. What is atopic dermatitis and how should it be defined in epidemiological studies? In: Williams HC, editor. Atopic dermatitis: the epidemiology, causes, and prevention of atopic eczema. Cambridge (UK): Cambridge University Press; 2000:3-24.

4. Kinross JM, Darzi AW, Nicholson JK. Gut microbiome-host interactions in health and disease. Genome Med 2011;3:14.
5. Bakhead F. Host responses to the human microbiome. Nutr Rev 2012;70:S14-7.

6. Cho I, Blaser MJ. The human microbiome: at the interface of health and disease. Nat Rev Genet 2012; 13:260-70.

7. Bjorksten B, Sepp E, Julge K, Voor T, Mikelsaar M. Allergy development and the intestinal microflora during the first year of life. J Allergy Clin Immun 2011;108:516-20.

8. Bottcher MF, Nordin EK, Sandin A, Midtvedt T, Bjorksten B. Microflora-associated characteristics in faeces from allergic and non-allergic infants. Clin Exper Allergy 2000;30:1591-6.

9. Isolauri E, Sutas Y, Kankaanpaa P, Arvilommi H, Salminen S. Probiotics: effects on immunity. Am J Clin Nutr 2001;73:444S-50S.

10. Kalliomaki M, Kirjavainen P, Eerola E, Kero P, Salminen S, Isolauri E. Distinct patterns of neonatal gut microflora in infants in whom atopy was and was not developing. J Allergy Clin Immun 2001;107: 129-34.

11. McKeever TM, Lewis SA, Smith C, Hubbard R. The importance of prenatal exposures on the development of allergic disease. Am J Respir Crit Care Med 2002;166:827-32.

12. Dom S, Droste JHJ, Sariachvili MA, et al. Pre- and post-natal exposure to antibiotics and the development of eczema, recurrent wheezing and atopic sensitization in children up to the age of 4 years. Clin Exp Allergy 2010;40:1378-87.

13. Alm JS, Swartz J, Lilja G, Scheynius A, Pershagen G. Atopy in children of families with an anthroposophic lifestyle. Lancet 1999;353:1485-8.

14. Droste JH, Wieringa MH, Weyler JJ, Nelen VJ, Vermoirc PA, Van Bever HP. Does the use of antibiotics in early childhood increase the risk of asthma and allergic disease? Clin Exp Allergy 2000;30:154753.

15. McKeever T, Lewis SA, Smith C, et al. Early exposure to infections and antibiotics and the incidence of allergic disease: a birth cohort study with the West Midlands General Practice Research Database. J Allergy Clin Immun 2002;109:43-50.

16. Negele K, Heinrich J, Borte M, et al.; LISA Study Group. Mode of delivery and development of atopic disease during the first 2 years of life. Pediatr Allergy Immunol 2004;15:48-54.

17. Falk PG, Hooper LV, Midtvedt T, Gordon JI. Creating and maintaining the gastrointestinal ecosystem: what we know and need to know from gnotobiology. Microbiol Mol Biol Rev 1998;62:1157-70.

18. Kero J, Gissler M, Gronlund M, et al. Mode of delivery and asthma - is there a connection? Pediatr Res 2002;52:6-11.

19. Hakansson S, Kallen K. Caesarean section increases the risk of hospital care in childhood for asthma and gastroenteritis. Clin Exp Allergy 2003;33:757-64. 
20. Cheng Q, Nelson D, Zhu S, Fishchetti VA. Removal of group B Streptococci colonizing the vagina and oropharynx of mice with a bacteriophage lytic enzyme. Antimcrob Agents Chemother 2005;49:111-7.

21. Easmon C, Hastings M, Deeley J, Bloxham B, Rivers R, Marwood R. The effect of intrapartum chemoprophylaxis on the vertical transmission of group $\mathrm{B}$ streptococci. Br J Obstet Gynaecol 1983;90:633-5.

22. Mackay G, House M, Bloch E, Wolfberg A. A GBS culture collected shortly after GBS prophylaxis may be inaccurate. J Matern Fetal Neonatal Med 2012; 25:736-8.

23. Penders J, Stobberingh EE, Thijs C, et al. Molecular fingerprinting of the intestinal microbiota of infants in whom atopic eczema was or was not developing. Clin Exp Allergy 2006;36:1602-8.

24. Farooqi IS, Hopkin JM. Early childhood infection and atopic disorder. Thorax 1998;53:927-32.

25. Dominguez-Bello MG, Costello EK, Contreras M, et al. Delivery mode shapes the acquisition and structure of the initial microbiota across multiple body habitats in newborns. Proc Natl Acad Sci U S A 2010;107:11971-5.

26. Bahrami B, Macfarlane S, Macfarlane GT. Induction of cytokine formation by human intestinal bacteria in gut epithelial lines. J Appl Microbiol 2010;110:353-63.

27. Verani JR, McGee L, Schrag SJ; Division of Bacterial Diseases, National Center for Immunization and Respiratory Diseases, Centers for Disease Control and Prevention (CDC). Prevention of perinatal group B streptococcal disease-revised guidelines from CDC, 2010. MMWR Recomm Rep 2010; 59(RR-10):1-36.

28. Lin FYC, Brenner RA, Johnson YR, et al. The effectiveness of risk-based intrapartum chemoprophylaxis for the prevention of early-onset neonatal group b streptococcal disease. Amer J Obstet Gynecol 2001;184:1204-10.

29. Moore MM, Rifas-Shiman SL, Rich-Edwards JW, et al. Perinatal predictors of atopic dermatitis occurring in the first six months of life. Pediatrics 2004;113: 468-74.

30. Strachan DP. Hay fever, hygiene, and household size. British Med J 1989;299:1259-60.

31. Rautava S, Ruuskanen O, Ouwehand A, Salminen S, Isolauri $\mathrm{E}$. The hygiene hypothesis of atopic disease - an extended version. J Pediatr Gastroenterol Nutr 2004;38:378-88.

32. Tessler R. Birth order, family size, and children's use of physician services. Health Serv Res 1979;3:55-62.

33. Chen AY, Escarce JJ. Effects of family structure on children's use of ambulatory visits and prescription medications. Health Serv Res 2006;41:1895-914.

34. Palmer CN, Irvine AD, Terron-Kwiatkowski A, et al. Common loss-of-function variants of the epidermal barrier protein filaggrin are a major predisposing factor for atopic dermatitis. Nat Genet 2006;38: 441-6.

35. Hanifin JM, Reed ML; Eczema Prevalence and Impact Working Group. A population-based survey of eczema prevalence in the United States. Dermatitis 2007;18:82-91. 